\title{
Pathomorphology and treatment of congenital anterolateral bowing of the tibia associated with duplication of the hallux
}

\author{
H. M. Manner, \\ C. Radler, \\ R. Ganger, \\ G. Grossbötzl, \\ G. Petje, \\ F. Grill
}

From the

Orthopaedic

Hospital Vienna-

Speising, Vienna, Austria

\footnotetext{
H. M. Manner, MD, Orthopaedic Resident C. Radler, MD, Orthopaedic Resident

R. Ganger, MD,

Orthopaedic Surgeon

G. Petje, MD, Orthopaedic

Surgeon

F. Grill, MD, Professor and

Head of Department

Paediatric Orthopedic

Department, Orthopaedic

Hospital Vienna-Speising,

Speisingerstrasse 109, 1130

Vienna, Austria.

G. Grossbötzl, MD,

Orthopaedic Surgeon

Department of Orthopaedic

Surgery, General Hospital

Linz, Krankenhausstrasse 9,

4020 Linz, Austria.

Correspondence should be sent to Dr H. M. Manner; e-mail:

hannes.manner@oss.at
}

C2005 British Editorial

Society of Bone and

Joint Surgery

doi:10.1302/0301-620X.87B2.

$15132 \$ 2.00$

$J$ Bone Joint Surg $[\mathrm{Br}]$

2005;87-B:226-30.

Received 12 November 2003;

Accepted after revision

6 January 2004

\begin{abstract}
Congenital unilateral anterolateral tibial bowing in combination with a bifid ipsilateral great toe is a very rare deformity which resembles the anterolateral tibial bowing that occurs in association with congenital pseudarthrosis of the tibia. However, spontaneous resolution of the deformity without operative treatment and with a continuously straight fibula has been described in all previously reported cases. We report three additional cases and discuss the options for treatment. We suggest that this is a specific entity within the field of anterolateral bowing of the tibia and conclude that it has a much better prognosis than congenital pseudarthrosis of the tibia, although conservative treatment alone may not be sufficient.
\end{abstract}

Pathological congenital bowing of the tibia in the newborn is rare. Posteromedial and anteromedial bowing may be treated by 'benign neglect', but anterolateral bowing should alert the surgeon to the possibility of the development of pseudarthrosis of the tibia and impending fracture. ${ }^{1,2}$ In contrast to other types of tibial bowing, anterolateral bowing in association with congenital tibial pseudarthrosis is rarely diagnosed at birth and usually develops during the first decade of life. ${ }^{2}$

In 1994, Tuncay, Johnston and Birch $^{3}$ reported five cases which seemed to represent a 'benign' form of congenital pseudarthrosis of the tibia. Anterolateral bowing of the tibia was observed, with the fibula remaining structurally normal and straight and the deformity of the tibia resolved spontaneously without any surgical treatment. Congenital anterolateral bowing of the tibia in association with a bifid ipsilateral great toe has been reported as a new entity. ${ }^{4-8}$ None of the reported cases was associated with neurofibromatosis or pseudarthrosis of the tibia. Spontaneous resolution of the axial deformity has been described. In all cases the anterolateral bowing was exclusively in the tibia, the fibula remaining straight and uninvolved.

In all reported cases the zone of irregularity in the tibia was limited to a small area at the apex of the anterolateral bow on the radiographs and did not continue distally or proximally. The pathomorphology of this area remains unknown. To our knowledge, hindand mid-foot deformities associated with this type of tibial bowing have not been described.
In previous reports, surgery was only performed for duplication of the ipsilateral great toe and metatarsal involvement. Leg-length discrepancy was noted by all previous authors but little attention was paid to persistent severe subluxation of the proximal tibiofibular joint due to the axial deformity and discrepancy of tibiofibular length.

We now report three further cases of severe anterolateral bowing of the tibia occurring in association with ipsilateral bifidity of the great toe.

\section{Patients and Methods}

We describe three patients (two girls and one boy) with congenital anterolateral bowing of the tibia in association with duplication of the hallux who presented between 1990 and 1999 . All three were between 14 and 29 days of age when first seen and all the deformities were unilateral. Pregnancy and birth histories were normal and family histories were negative for deformities of the lower limb and neurofibromatosis. No other deformities were present at birth or developed later.

All three patients had the same deformities. At birth the tibia was markedly bowed in the anterolateral direction. The apex of the deformity was in the middle of the diaphysis of the tibia. Plain radiography showed some irregularity which suggested localised duplication of the tibial medullary canal. Duplication of the great toe was present in all three patients and treated by early resection and reconstruction during the first year of life (Fig. 1). 


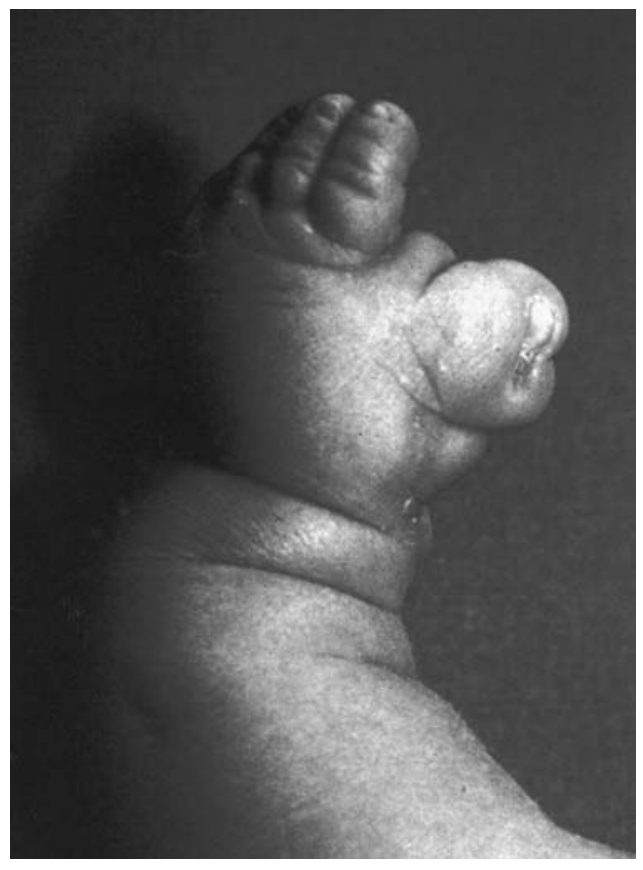

Fig. 1

Photograph of duplication of the great toe at birth.

During the first four years of life the tibial deformity spontaneously corrected to some extent. Despite treatment by orthoses after the age of four years, further spontaneous resolution of the tibial bow was not seen. A leg-length discrepancy up to $5.5 \mathrm{~cm}$ also developed. The area of major tibial bowing was found in the middle third of the diaphysis in all three patients. Plain radiography and MRI showed signs of focal tibial duplication in the area of the bowing (Fig. 2). No signs of pseudarthrosis or other clinical or radiological features suggestive of neurofibromatosis were seen. The fibula appeared to be structurally normal and straight but clinically the fibula head was prominent prox- imally causing recurrent localised pain due to overgrowth of the fibula and subluxation of the proximal tibiofibular joint (Fig. 3) (Table I).

Reconstructive surgery using the Ilizarov method was performed at the age of five to six years. The aim was to correct the leg-length discrepancy with simultaneous axial correction and treatment of the subluxation of the proximal tibiofibular joint. All patients were treated by the same step-by-step treatment. The Ilizarov ring fixator was applied, and tibial osteotomy performed at the site of maximal tibial bowing.

Lengthening of the tibia alone, without fibular osteotomy, allowed gradual distalisation of the fibular head. After completion of step one, gradual correction of the bowing and simultaneous further lengthening were performed. Slight overlengthening was carried out to anticipate further leg-length discrepancy with growth (Table II).

Illustrative case report. A five-year-old girl was born at 42 weeks of gestation to non-consanguineous parents. The family history was negative for neurofibromatosis and bowing of the tibia. At birth severe anterolateral bowing of the midshaft and shortening of the right tibia compared with the ipsilateral fibula and contralateral tibia were present. Ipsilateral hexadactyly with duplication of the great toe was evident clinically and radiologically and led to severe foot problems. Surgery was first performed at the age of one year when the duplicated medial anlage of the great toe was resected. Metatarsal hypoplasia and duplication of the medial cuneiform and navicular led to marked shortening of the first ray. The tibial bowing was treated by an orthosis until the age of five years. Partial but not complete spontaneous resolution of the tibial malalignment was seen.

At the age of five years the indications for operative reconstructive surgery were severe proximal fibular overgrowth and gait problems because of marked varus of the ankle and leg-length discrepancy. Plain radiography showed malalignment of $20^{\circ}$ in the frontal and $5^{\circ}$ in the
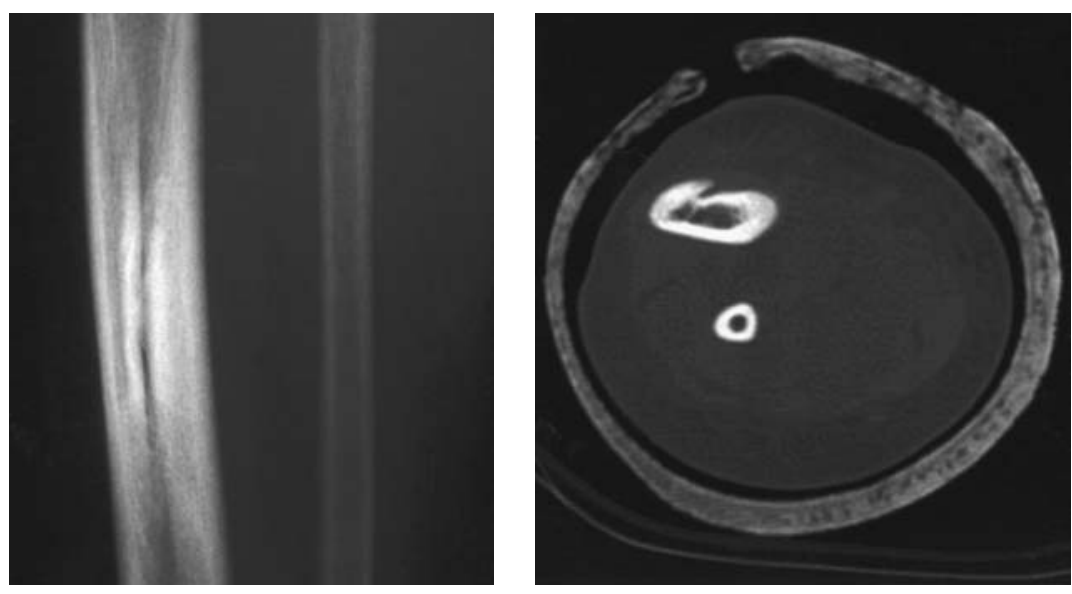

Fig. 2a
Fig. $2 b$
Signs of a focal duplication of the medullary canal of the tibia on a) plain radiography and b) MRI. 


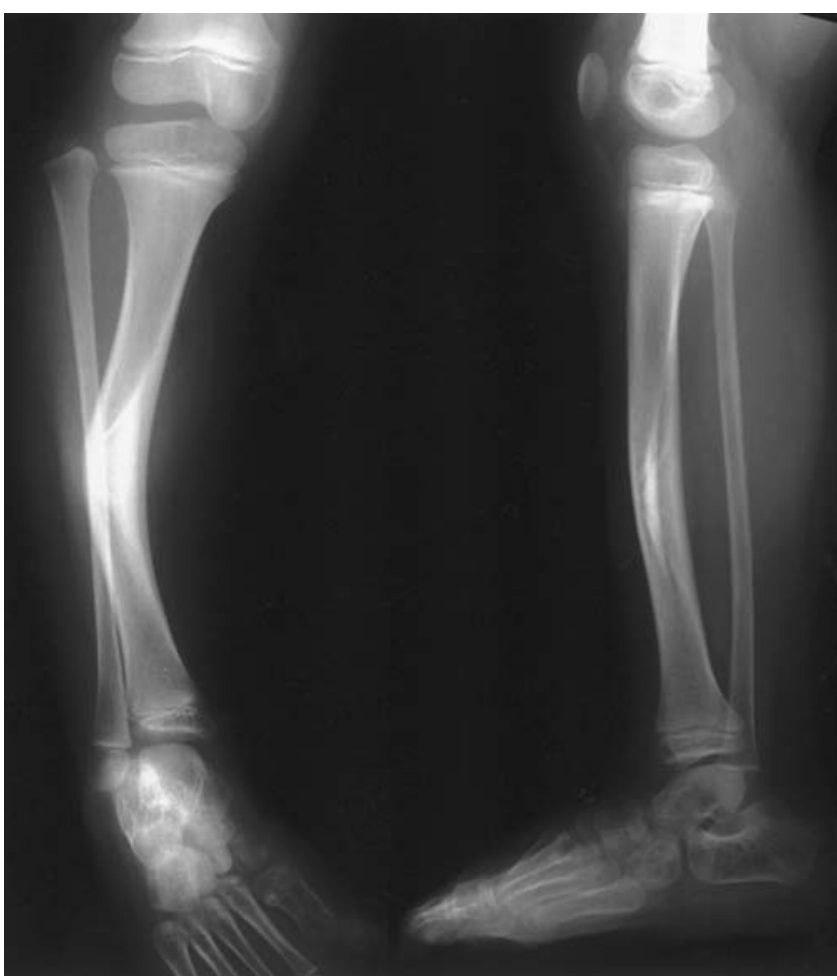

Fig. 3

Anteroposterior and lateral radiographs showing overgrowth of the proximal fibula at five years of age.

sagittal plane. Another striking feature was the marked posteromedial thickening of the tibial cortical bone, with no sign of cystic or degenerative change suggestive of congenital tibial pseudarthrosis. MRI showed signs of tibial duplication. The tibial medullary canal was continuously open but narrow in the area of the bowing. The distal tibial epiphyseal height was $0.6 \mathrm{~cm}$ compared with $0.9 \mathrm{~cm}$ on the contralateral side and the distal tibial epiphyseal width was
$3.1 \mathrm{~cm}$ compared with $3.6 \mathrm{~cm}$ on the contralateral side suggesting slight tibial hypoplasia. Orthoradiography showed a deficiency of tibial length of $17 \%$ compared with the contralateral side, and a leg-length discrepancy of $3.1 \mathrm{~cm}$. The fibula was straight and showed no pathological bony findings suggestive of pseudarthrosis. Proximal overgrowth in comparison with the ipsilateral tibia was $2.8 \mathrm{~cm}$, leading to subluxation of the proximal tibiofibular joint. The fibula was hyperplastic distally.

Treatment using the Ilizarov external fixator was undertaken in two steps. Initially, tibial osteotomy in the diaphyseal area with minimal partial resection and partial acute correction was performed. Intra-operatively, a well-defined duplication of the medullary canal of the tibia was seen (Fig. 4). At the same operation, proximal tibial percutaneous osteotomy was performed to allow tibial lengthening and simultaneous lowering of the fibular head with distal fixation only of the fibula to the frame. After lengthening of the proximal tibia of $2.6 \mathrm{~cm}$, the fibular head had migrated to its correct anatomical position. The second step of the procedure was then undertaken fixing the fibula to the proximal ring with a fine wire together with midshaft fibular osteotomy to allow further tibial lengthening and axial correction. Distraction was completed with slight overlengthening $(0.7 \mathrm{~cm})$ and complete correction of the axial deformity. After full consolidation of the lengthened bone, the apparatus was removed at 140 days with a healing index of 37 days per $\mathrm{cm}$. At the latest follow-up six years after surgery, no recurrent tibial deformity was present but the duplication of the navicular, cuneiform, and first metatarsal bones were causing pain and difficulties with gait (Fig. 5).

\section{Results}

The results of reconstructive surgery in the three patients are summarised in Table II.

The fibula remained straight during axial correction and lengthening of the tibia, with no sign of thinning or cystic

Table I. Radiological measurements showing spontaneous relief of the anterolateral bow whereas leg-length discrepancy and proximal overlength of the fibula are severe

\begin{tabular}{llllll}
\hline Case & $\begin{array}{l}\text { Age at surgery } \\
\text { (yrs) }\end{array}$ & $\begin{array}{l}\text { Lateral bow at } \\
\text { birth/at surgery }\left(^{\circ}\right)\end{array}$ & $\begin{array}{l}\text { Anterior bow at } \\
\text { birth/at surgery }\left(^{\circ}\right)\end{array}$ & $\begin{array}{l}\text { LLD }^{*} \text { at surgery } \\
\text { (cm) }\end{array}$ & $\begin{array}{l}\text { Proximal overlength } \\
\text { of fibula (cm) }\end{array}$ \\
\hline 1 & 6 & $45 / 22$ & $40 / 15$ & 5.5 & 2.3 \\
2 & 5 & $45 / 15$ & $38 / 14$ & 4.4 & 1.8 \\
3 & 5 & $60 / 23$ & $60 / 5$ & 3.1 & 2.8 \\
\hline * leg-length discrepancy
\end{tabular}

Table II. The end results of operative lengthening of the tibia show that slight overlengthening was achieved. The healing indices were within the normal range

\begin{tabular}{lllllll}
\hline Case & $\begin{array}{l}\text { LLD* at surgery } \\
(\mathbf{c m})\end{array}$ & $\begin{array}{l}\text { Amount of lengthening } \\
(\mathbf{c m})\end{array}$ & $\begin{array}{l}\text { Resulting overlength } \\
(\mathbf{c m})\end{array}$ & Days in frame & $\begin{array}{l}\text { Healing index } \\
\text { (days/cm) }\end{array}$ & $\begin{array}{l}\text { Recurrence of deformity } \\
\text { at follow-up }\end{array}$ \\
\hline 1 & 5.5 & 6.5 & 1.0 & 273 & 42 & No \\
2 & 4.4 & 5.0 & 0.6 & 205 & 41 & No \\
3 & 3.1 & 3.8 & 0.7 & 140 & 37 & No \\
\hline
\end{tabular}

* leg-length discrepancy 


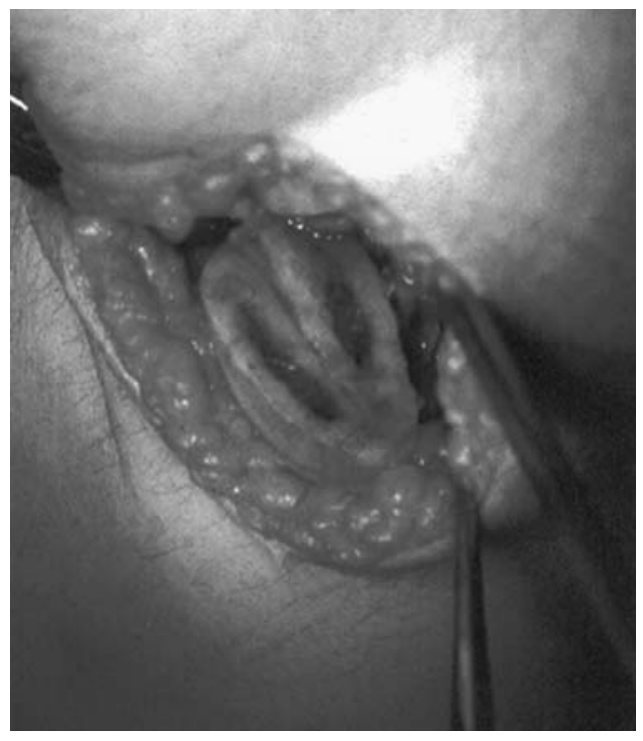

Fig. 4

Photograph of a clear tibial duplication during osteotomy in the area of the major bowing.

degeneration. Leg-length discrepancy and axial deviation were completely corrected. The healing indices were within the normal range and there was no sign of recurrent deformity at follow-up. However, foot pressure and problems with the wearing of shoes persisted because of metatarsal and tarsal abnormalities on the medial side of the foot.

\section{Discussion}

Congenital anterolateral bowing of the tibia is a rare deformity reported to occur in one in 140000 live births. ${ }^{9}$ In 1949, Heyman and Herndon ${ }^{10}$ classified tibial bowing according to the direction of the deformity. Although congenital anteromedial and posteromedial tibial bowing tends to resolve spontaneously during growth, ${ }^{11,12}$ anterolateral bowing is a more serious deformity. Congenital pseudarthrosis of the tibia is rarely observed at birth. It usually develops during the first decade of life $^{2}$ and may present as a fracture through the highly sclerotic or atrophic bone in the tibia. ${ }^{1}$ Involvement of the fibular bone in the deformity is reported to occur in $62 \%$ of cases and can be important in the diagnosis of congenital pseudarthrosis of the tibia when the radiological findings in the tibia are inconclusive. $^{13}$

Congenital anterolateral tibial bowing with spontaneous resolution of the deformity with ${ }^{14,15}$ or without ${ }^{3,16}$ involvement of the fibula has been reported. Medial endosteal thickening extending across the tibial bow has commonly been reported. None of our patients showed cystic, extensive sclerotic, or other degenerative signs. No associated deformities of the foot have been reported.

The association of ipsilateral duplication of the hallux with anterolateral tibial bowing was first reported by Kardon et $\mathrm{al}^{5}$ in 1986. Subsequently, Adamsbaum et $\mathrm{al}^{7}$ Weaver et $\mathrm{al}^{8}{ }^{8}$ Bressers and Castelein ${ }^{4}$ and Kitoh et $\mathrm{al}^{6}$ added similar cases with greater detail of the radiological changes in the tibia. In cases of anterolateral tibial bowing without associated deformities of the foot, reactive posteromedial endosteal thickening was commonly reported. The

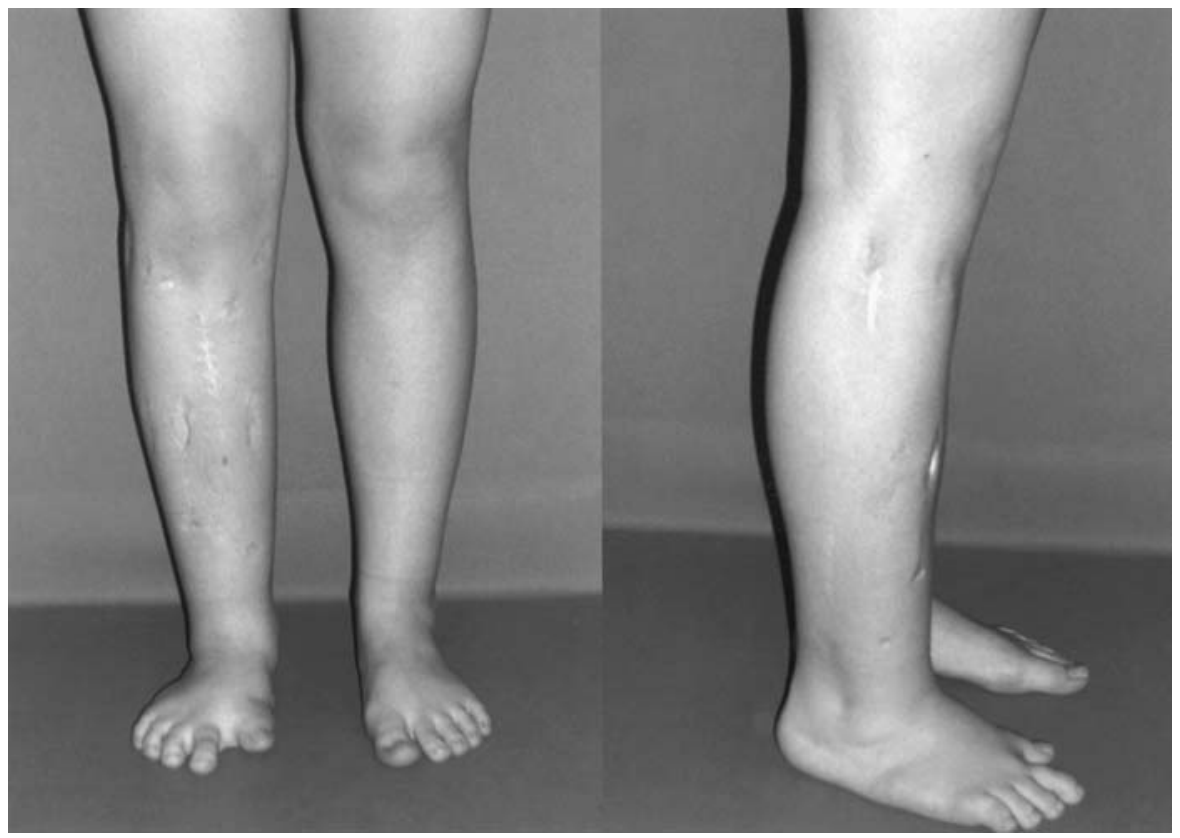

Fig. 5 a

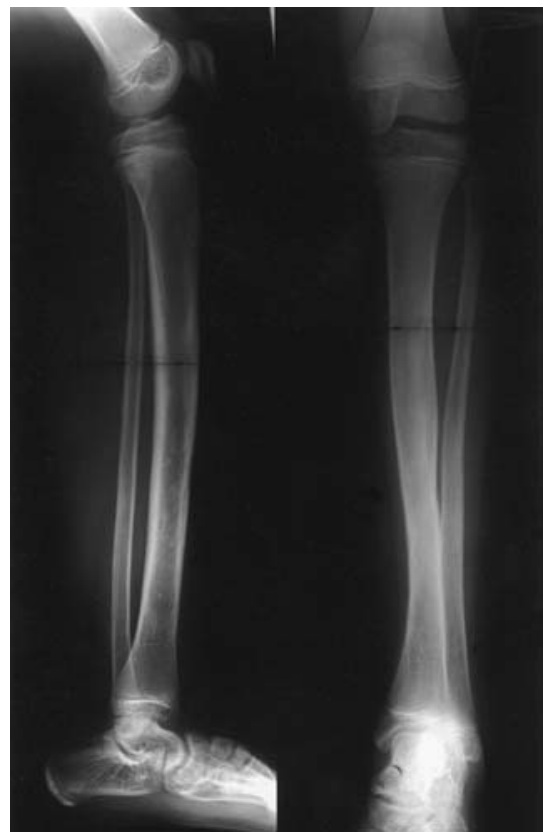

Fig. 5b

Outcome of case 3 at last follow-up a) clinically and b) radiologically. 
deformity was noted to be most severe at birth and spontaneous resolution occurred with growth. Nevertheless, the apex of the tibial deformity showed some irregularity. Watanabe, Fujita and $\mathrm{Oka}^{17}$ and Adamsbaum et $\mathrm{al}^{7}$ reported a short incomplete tibial duplication based on their CT findings. Weaver et $\mathrm{al}^{8}$ and Bressers and Castelein ${ }^{4}$ thought that the radiological signs of duplication were the result of remedullarisation of the tibial canal. Other authors observed a partial cleft in the tibia in the area of the bow on $\mathrm{CT}^{6,7,15}$ and suspected incomplete duplication. In the long term, this area of uncertainty at the apex of the tibial bow disappears during resolution of the bow. In our patients MRI revealed a partial cleft, and plain radiography showed possible tibial duplication, but could not confirm true duplication. However, during surgery, true tibial duplication was seen with two distinct cortices surrounding both tibial medullary canals (Fig. 4). This pattern of duplication regressed during growth, and a single well-defined medullary canal developed.

In our cases as in all previous reports of congenital anterolateral tibial bowing in association with duplication of the hallux, the fibula was not involved. ${ }^{4,6,8}$ It was straight and appeared structurally uninvolved in the pathomorphological changes affecting the tibia and foot.

Proximal overgrowth of the fibula compared with the ipsilateral tibia led to subluxation of the proximal tibiofibular joint but overall the fibula was slightly shorter than the contralateral fibula. This may be related to ligamentous tethering due to the relative shortening of the tibia.

In our three patients, the tibia was hypoplastic distally with a diminished width of the epiphysis. The association of preaxial polydactyly and tibial deficiencies is well known. ${ }^{18}$ Lewin and Opitz ${ }^{19}$ described a tibial and fibular field during the development of the lower limb. The tibial field involved the distal femur, tibia, and preaxial toes.

With regard to previous reports of associated foot deformities, our cases showed similar duplication of the hallux. We agree with other authors that surgical treatment of this duplication is difficult. ${ }^{8}$ Foot problems persisted in our patients because of pes planus and severe shortening of the first ray. Our patients also had persistent problems with shoe wear due to widening of the midfoot associated with partial duplication of the navicular, cuneiform, and metatarsal bones. This deformity has not been previously described but may be typical of developmental disorders affecting the tibial field.

Previous authors have recommended conservative treatment since spontaneous complete resolution of the anterolateral bowing during growth can occur.

However, this does not take account of the leg-length discrepancy and subluxation of the proximal tibiofibular joint. In our patients, spontaneous resolution of the tibial bow was observed to occur during the first four years of life, but then persisted to a certain degree leading to foot problems. All our patients complained of intermittent pain in the foot and at the proximal tibiofibular joint before surgery. Reconstructive surgery was indicated for a leg-length discrepancy of up to $5.5 \mathrm{~cm}$, persistent anterolateral bowing and proximal subluxation of the fibula. Even when corrective osteotomy was performed in the area of the bow, healing indices were comparable with those in the literature for lengthening procedures in structurally normal bone. The deformity had not recurred at the latest follow-up, but intermittent pain in the foot persisted. We are therefore in agreement with Weaver et $\mathrm{al}^{8}$ that the deformity of the tibia is relatively benign whereas the deformity of the foot is not and may be the most important factor affecting the longterm outcome in this condition.

No benefits in any form have been received or will be received from a commercial party related directly or indirectly to the subject of this article.

\section{References}

1. Grill F, Bollini G, Dungle P, et al. Treatment approaches for congenital pseudarthrosis of tibia: results of the EPOS multicenter study. J Pediatr Orthop B2000;9:75-89.

2. Hefti F, Bollini G, Dungl P, et al. Congenital pseudarthrosis of the tibia: history, etiology, classification, and epidemiologic data. J Pediatr Orthop B 2000;9:11-15.

3. Tuncay IC, Johnston CE 2nd, Birch JG. Spontaneous resolution of congenital anterolateral bowing of the tibia. J Pediatr Orthop 1994;14:599-602.

4. Bressers MM, Castelein RM. Anterolateral tibial bowing and duplication of the hallux: a rare but distinct entity with good prognosis. J Pediatr Orthop B 2001;10:153-7.

5. Kardon NB, Dana LP, Fitzgerald JM, Opitz JM. Two sporadic cases of amelia/ phocomelia with similar phenotype: rare and unusually symmetrical form of FFR dysostosis or separate entity? Am J Med Genet Supp/ 1986;2:239-45.

6. Kitoh H, Nogami H, Hattori T. Congenital anterolateral bowing of the tibia with ipsilateral polydactyly of the great toe. Am J Med Genet 1997;73:404-7.

7. Adamsbaum C, Kalifa G, Seringe R, Bonnet JC. Minor tibial duplication: a new cause of congenital bowing of the tibia. Pediatr Radiol 1991;21:185-8.

8. Weaver KM, Henry GW, Reinker KA. Unilateral duplication of the great toe with anterolateral tibial bowing. J Pediatr Orthop 1996;16:73-7.

9. Andersen KS, Bohr H, Sneppen O. Congenital angulation of the lower leg: crus curvatum congenitum. Acta Orthop Scand 1968;39:387-97.

10. Heyman $\mathbf{C H}$, Herndon $\mathbf{C H}$. Congenital posterior angulation of the tibia. J Bone Joint Surg [Am] 1949;31-A:571-80.

11. Hofmann A, Wenger DR. Posteromedial bowing of the tibia: progression of discrepancy in leg lengths. J Bone Joint Surg [Am] 1981;63-A:384-8.

12. Pappas AM. Congenital posteromedial bowing of the tibia and fibula. J Pediatr Orthop 1984; 4:525-31.

13. Keret $\mathbf{D}$, Bollini $\mathbf{G}$, Dungle $\mathbf{P}$, et al. The fibula in congenital pseudoarthrosis of the tibia: the EPOS multicenter study. J Pediatr Orthop B 2000;9:69-74.

14. Newell RL, Burbin FC. The aetiology of congenital angulation of tubular bones with constriction of the medullary canal, and its relationship to congenital pseudarthrosis. J Bone Joint Surg [Br] 1976;58-B:444-7.

15. Kaneda M, Teramoto S, Shirasu Y. Anatomical features associated with preaxial duplication (pd): a recessive mutation in the rat. Teratology 1989;40:77-84.

16. Badgley CE, O'Connor SJ, Kudner DF. Congenital kyphoscoliotic tibia. J Bone Joint Surg [Am] 1952;34-A:349-69.

17. Watanabe H, Fujita S, Oka I. Polydactyly of the foot: an analysis of 265 cases and a morphological classification. Plast Reconstr Surg 1992;89:856-77.

18. Pavone L, Viljoen D, Ardito S, et al. Two rare developmental defects of the lower limbs with confirmation of the Lewin and Opitz hypothesis on the fibular and tibial developmental fields. Am J Med Genet 1989;33:161-4.

19. Lewin SO, Opitz JM. Fibular a/hypoplasia review and documentation of the fibular developmental field. Am J Med Genet Supp/1986;2:215-38. 